\title{
MEMS FORCE PROBES FOR CELL MECHANOBIOLOGY AT THE MICROSECOND SCALE
}

\author{
J.C. Doll and B.L. Pruitt* \\ Stanford University, Stanford, California, USA
}

\begin{abstract}
We present high-speed force probes with on-chip actuation and sensing for the measurement of $\mathrm{pN}$-scale forces at the microsecond time scale. We achieve a high resonant frequency in water (1-200 kHz) with requisite low spring constants $(0.3-40$ $\mathrm{pN} / \mathrm{nm})$ and low integrated force noise $(1-100 \mathrm{pN})$ by targeting probe dimensions on the order of $300 \mathrm{~nm}$ thick, 1-2 $\mu \mathrm{m}$ wide and 30-200 $\mu \mathrm{m}$ long. Forces are measured using silicon piezoresistors while the probe is actuated thermally.
\end{abstract}

\section{INTRODUCTION}

A variety of instruments have been developed for the precise measurement of forces and displacements in the past 25 years. The most commonly used techniques are atomic force microscopy, magnetic tweezers, and optical tweezers. These tools are capable of measuring force $\left(10^{-14}-10^{-8} \mathrm{~N}\right)$, displacement $\left(10^{-10}-10^{-4} \mathrm{~m}\right)$ and time $\left(10^{-4}-10^{2} \mathrm{sec}\right)$ over many orders of magnitude [1]. However, none are capable of simultaneously measuring and delivering $\mathrm{pN}$ scale forces with microsecond time resolution.

The ideal force probe needs to have comparable stiffness to the system of interest (e.g. $0.1-10 \mathrm{pN} / \mathrm{nm}$ for cells and molecules) while maintaining a high resonant frequency during operation in liquid (e.g. $100 \mathrm{kHz}$ ). Optical and magnetic tweezers can be built with vanishingly small spring constants (e.g. $10^{-3} \mathrm{pN} / \mathrm{nm}$ ), but the relatively large mass of the bead limits their bandwidth to 1-10 $\mathrm{kHz}$. Microcantilevers have been fabricated with measurement bandwidths of over $5 \mathrm{MHz}$ in water, but their spring constants have been correspondingly large, again due to their relatively large size.

In order to address this technological gap, we have developed force probes with on-chip piezoresistive force readout and thermal actuation. A cantilever beam structure minimizes the probe mass while allowing simple spring constant tuning via the length of the probe. Microcantilevers with integrated sensing and actuation have been reported previously [2-4]. However, they were designed for high-speed scanning probe microscopy, so were relatively large (e.g. $100 \mu \mathrm{m}$ wide x $3 \mu \mathrm{m}$ thick) and optimized for displacement resolution at the expense of force resolution. In contrast, our devices are optimized for single point force measurements and are 10-100x smaller than prior integrated devices.

\section{DESIGN}

The force probe topology is presented in Figure 1. The probe consists of a narrow, soft sensor at the tip and a wide, stiff actuator at the base. We have designed and fabricated two different probe varieties: sensor-only devices (PR) and thermally actuated devices (PRT). The PR devices do not include an actuator but retain the stiffened base in order to define the force probe length from the front of the wafer rather than during the imprecise backside release etch. Placement of the stiff, heavy actuator at the base of the probe maximizes the tip deflection range, maximizes the overall resonant frequency of the probe, minimizes the thermal actuator time constant and decouples the mechanical design of the sensor and actuator portions.

The piezoresistor is designed using numerical optimization [5]. Although the piezoresistor design problem is not convex, seeding the numerical optimizer with several random initial guesses leads to a globally optimal design. Our design optimization code now captures effects such as self-heating in piezoresistive cantilevers [6] and integrates thermal modeling and temperature constraints. The piezoresistors are designed to minimize the RMS force noise in the intended measurement bandwidth while constraining the increases in the maximum and tip temperatures to approximately 5 and $1 \mathrm{~K}$ during operation in water, respectively.

The tradeoff between spring constant and resonant frequency improves as the size of the force probe decreases. A cantilever beam thickness of $300 \mathrm{~nm}$ was chosen due to the rapid reduction in thermal conductivity and uncertainty in the effective elastic modulus with any further reduction in thickness. But a thin device requires a shallow piezoresistor. The open source modeling and optimization code that we developed handles diffused, epitaxial and ion implanted piezoresistors (via lookup tables). We opted to fabricate the piezoresistors using $\mathrm{POCl}_{3}$ predeposition due to the relatively simple fabrication process and higher performance of $n$ type compared with p-type piezoresistors for this application [5]. Metal interconnects connect the piezoresistor to the bondpads, minimizing both the excess interconnect resistance and mechanical crosstalk during simultaneous actuation and sensing [3].

The actuator consists of a silicon heater, $\mathrm{SiO}_{2}$ etch stop and a layer of $\mathrm{Al}$. The heater increases the temperature of the actuator region, deflecting the probe tip due to the mismatch in thermal expansion coefficients between $\mathrm{Si}(2.6 \mathrm{ppm} / \mathrm{K})$ and $\mathrm{Al}(23 \mathrm{ppm} / \mathrm{K})$. The $\mathrm{SiO}_{2}$ thickness $(100 \mathrm{~nm})$ is chosen to minimize the thermal resistance between the heater and $\mathrm{Al}$ while providing an adequate etch stop. The thickness of the Al layer $(1000 \mathrm{~nm})$ is chosen to place the neutral axis at the $\mathrm{SiO}_{2}-\mathrm{Al}$ interface. The length of the actuator portion is a tradeoff between the heater time constant,

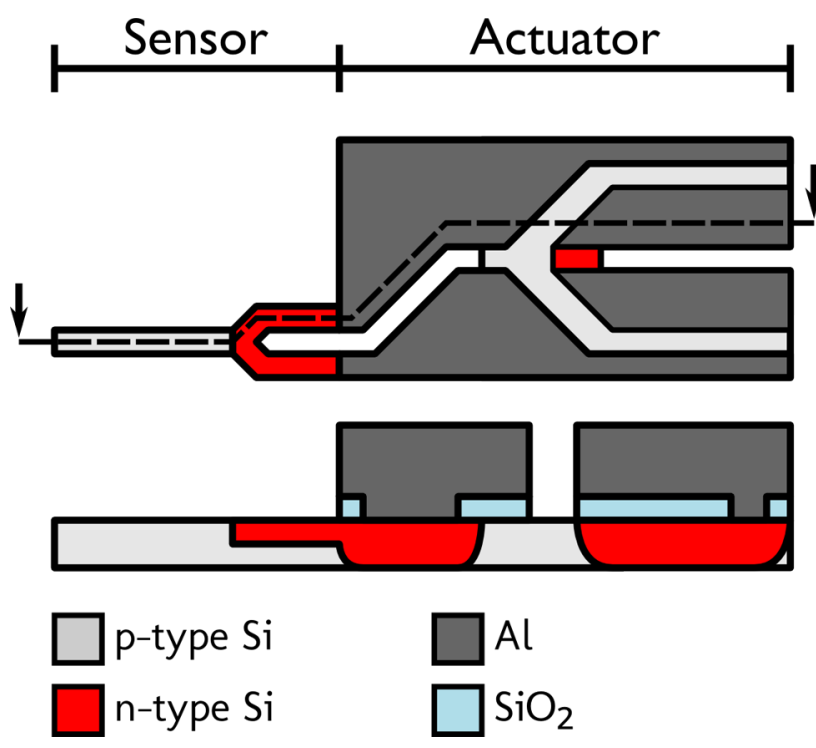

Figure 1: Layout of a force probe with integrated piezoresistive sensing and thermal actuation (PRT). The probe consists of a narrow, soft sensor attached to the end of a wide, stiff actuator. Placement of the actuator at the base rather than the tip leads to numerous performance benefits and decouples the mechanical and thermal design of the actuator and sensor.

Solid-State Sensors, Actuators, and Microsystems Workshop Hilton Head Island, South Carolina, June 3-7, 2012 
overall resonant frequency of the probe, and the maximum tip deflection. The overall resonant frequency of the probe is modeled using both finite element analysis and numerically using the Rayleigh-Ritz method. The target maximum tip deflection for the devices is $500 \mathrm{~nm}$ for the study of mammalian cochlear hair cells.

\section{FABRICATION}

The seven mask fabrication process is illustrated in Figure 2. The PR and PRT probes are fabricated using the same process and use identical film thicknesses.

The process starts with a (100) oriented $100 \mathrm{~mm}$ diameter silicon-on-insulator (SOI) wafer with a $340 \mathrm{~nm}$ thick device layer, $400 \mathrm{~nm}$ thick buried oxide (BOX) and $400 \mu \mathrm{m}$ thick handle wafer. The device layer is p-type silicon with a resistivity of 10-20 $\Omega$-cm. Poly-on-insulator (POI) wafers with comparable device and BOX layer thicknesses were fabricated and processed alongside the SOI wafers for process development. All lithography steps are performed using $0.7 \mu \mathrm{m}$ thick SPR955 (unless otherwise noted) on an ASML PAS 5500/60 stepper with backside alignment capability. The critical dimensions and overlay tolerances of the process are $0.5 \mu \mathrm{m}$ and $0.25 \mu \mathrm{m}$, respectively.

First, alignment marks are patterned and etched $120 \mathrm{~nm}$ into the device layer. The wafer is rotated by 45 degrees during the alignment mark exposure so that the piezoresistors will be oriented in the $<100>$ direction $( \pm 0.5$ degrees as set by the device layer orientation tolerance).

The oxide diffusion mask is formed next. The wafers are diffusion cleaned and a $90 \mathrm{~nm}$ thick thermal oxide layer is grown by oxidizing them in steam at $900 \mathrm{C}$. The thermal oxidation decreases the thickness of the device layer from 340 to the target thickness of $300 \mathrm{~nm}$. An additional $320 \mathrm{~nm}$ thick layer of low temperature oxide (LTO) is deposited on the wafers at $400 \mathrm{C}$ in order to increase the thickness of the oxide mask and prevent the diffusion of phosphorus through the oxide mask during the high temperature contact diffusion. After the LTO deposition the wafers are annealed in $\mathrm{N}_{2}$ at $1100 \mathrm{C}$ for 20 hours in order to densify the LTO and noticeably reduces strain gradients in the device layer.

The piezoresistive sensor is formed using two separate diffusions. First, the piezoresistor contacts are $\mathrm{POCl}_{3}$ doped at $900 \mathrm{C}$ for 20 minutes. Afterwards the thin layer of phosphosilicate glass (PSG) deposited on the surface is stripped in a dilute 50:1 HF solution, preventing undercut during the next step while leaving the underlying oxide mask intact. In the second diffusion the window in the oxide diffusion mask is opened further to dope the entire piezoresistor region at $825 \mathrm{C}$ for 30 minutes. The oxide windows are wet etched in 20:1 buffered oxide etchant (BOE) after the photoresist has been rendered hydrophilic in a $60 \mathrm{sec}$ low temperature $\mathrm{O}_{2}$ plasma pretreatment step. The high surface phosphorus concentration leads to slight upward device curvature. Minimizing the piezoresistor length reduces both the static tip deflection and the tip temperature increase during operation.

All of the remaining oxide is stripped and the device layer is patterned and etched via reactive ion etching (RIE). Afterwards the wafer is cleaned, $100 \mathrm{~nm}$ of LTO is deposited and oxide vias are patterned and wet etched. As in the rest of the oxide etch steps the wafer is pretreated with $\mathrm{O}_{2}$ plasma in order to render the photoresist hydrophilic and the vias are etched using 20:1 BOE. The etch is timed by visually monitoring the backside of the wafer and over etching by $20 \%$. After the wafers are cleaned in PRS1000 , the native oxide is removed with a brief 50:1 HF dip before rushing the wafers to the sputterer load lock for metal deposition.

In the sputterer load lock the wafers are heated to $200 \mathrm{C}$ for 3 minutes using quartz lamps to drive off any moisture before depositing $50 \mathrm{~nm}$ thick Ti and $1000 \mathrm{~nm}$ thick Al layer. The Ti layer provides a lower barrier height than Al to n-type silicon ( 0.5 vs 0.7 $\mathrm{eV}$ ) and serves as a contact diffusion barrier. The metal is lithographically patterned and etched back via RIE, stopping on the LTO layer. Afterwards the wafers are rinsed in water to eliminate residual $\mathrm{HCl}$ on the wafer before stripping the photoresist mask. Next a $400 \mathrm{~nm}$ thick LTO layer is deposited on the wafer and a trench is patterned and etched via RIE into the oxide around the force probe. The trench prevents cracks in the BOX from snapping the force probes, and increases probe yield from about $35 \%$ to $70 \%$ (and up to $90 \%$ ) [7].

The backside of the wafer is patterned with a $7 \mu \mathrm{m}$ thick layer of SPR220 and baked for two hours at 90C to harden it. The frontside of the wafer is left unprotected in order to maximize heat transfer during the backside DRIE. The wafers are shipped to the Lurie Nanofabrication Facility (LNF) at the University of Michigan for the backside etch. A staff member at the LNF bonds the device wafer to a carrier wafer using CrystalBond 555 (CB) using a custom bonding tool that eliminates air pockets in the $\mathrm{CB}$. After the wafers are etched and shipped back, the backside photoresist is stripped and the wafer is debonded from the carrier by soaking in $70 \mathrm{C}$ water for 30 minutes. The LTO coating deposited earlier prevents metal corrosion during this step. Afterwards the wafer is cleaned in fresh DI water and air dried.

The wafers are cleaned in a downstream $\mathrm{O}_{2}$ plasma asher to remove residual DRIE passivation polymer. However a thin layer of fluorocarbon polymer remains on the wafer because it is not volatile in pure $\mathrm{O}_{2}$ plasma. Without further cleaning a thin web of polymer remains on the wafer, rendering most of the devices unusable. A second polymer cleanup step is performed in a $\mathrm{CF}_{4} / \mathrm{O}_{2}$ plasma in order to remove the remaining fluorocarbon polymer. (a)

(b)

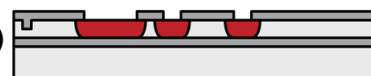

(c)

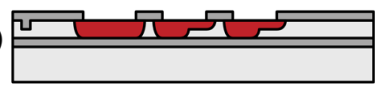

(d)

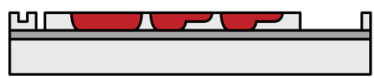

(e)

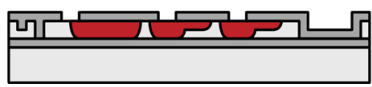

(f)

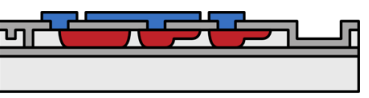

(g)

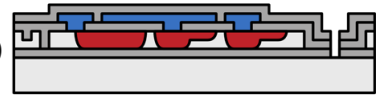

(h)

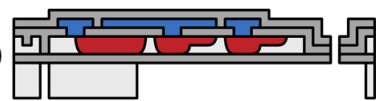

(i)

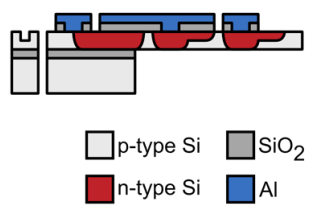

Figure 2: Fabrication process for force probes with integrated piezoresistive sensing and thermal actuation (PRT). (a) Alignment marks are patterned and etched. (b) The wafers are oxidized, coated with additional low temperature oxide (LTO) and annealed. Windows are opened in the oxide mask and the contacts are $\mathrm{POCl}_{3}$ doped at 900C. (c) The oxide windows are opened further and the piezoresistors are $\mathrm{POCl}_{3}$ doped at $825 \mathrm{C}$. (d) The oxide is stripped and the device layer is patterned and etched via reactive ion etching (RIE). (e) A layer of LTO is deposited and vias are opened before (f) the Ti/Al metal stack is deposited and patterned via RIE. (g) An additional layer of LTO is deposited and cracks are patterned into the oxide stack in order to increase device yield. (h) The backside of the wafer is patterned, bonded to a carrier wafer, etched via DRIE and debonded. (i) The BOX and LTO layers are etched via vapor HF in order to release the probes. 
Finally, the BOX and LTO layers are etched in vapor HF in order to release the force probes. The wafers are etched from the frontside, but cracks in the BOX allow the vapor HF to attack the BOX and LTO layers from both sides at once. The top LTO coating is approximately the same thickness as the BOX and minimizes undercut of the LTO layer underneath the metal interconnects. No forming gas anneal (FGA) is used because we found that it has a detrimental effect on the contact resistivity, even with the Ti diffusion barrier, due to the shallow piezoresistors.

Scanning electron micrographs (SEMs) of finished PR and PRT devices are presented in Figure 3. After cleanroom fabrication is complete, the devices are snapped out of the wafer using snap tabs defined during the backside DRIE step. The devices are epoxied to custom printed circuit boards (PCBs) and ultrasonically wirebonded with Al wire. Afterwards the wirebonds are coated with epoxy and the entire assembly is coated with a 200-300 nm thick layer of parylene N. Passivation is required for operation in water but is not necessary for experiments in air or vacuum.


Figure 3: Scanning electron micrographs (SEMs) of finished (a) PR and (b) PRT force probes.
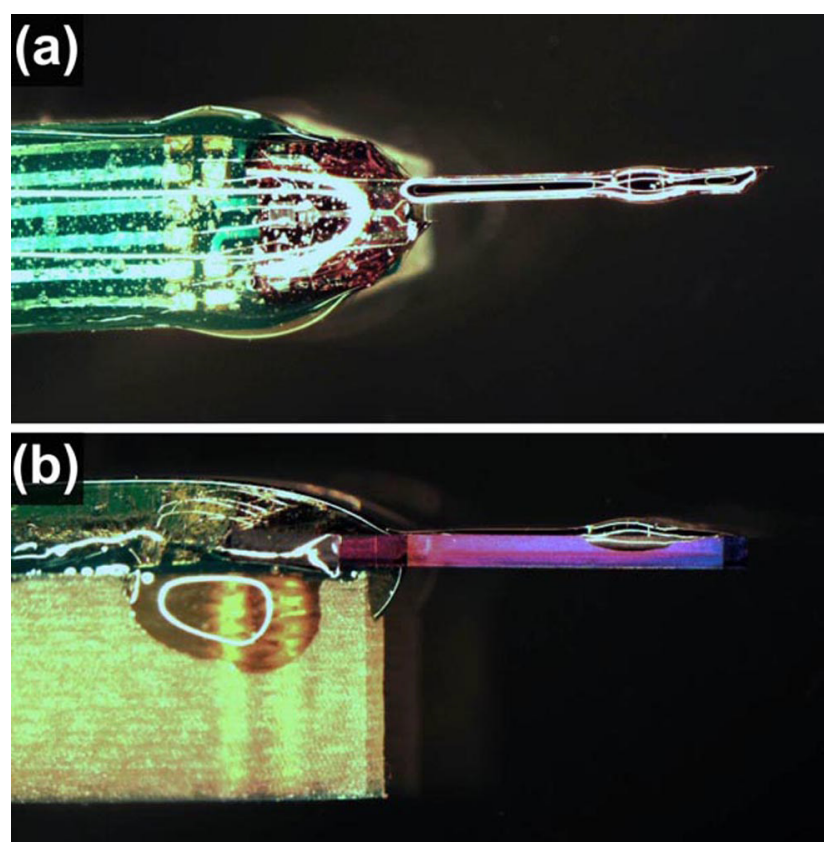

Figure 4: (a) Top and (b) side views of a fully assembled and passivated device. First, the silicon die is epoxied and wirebonded to the PCB. Next, the wirebonds and long silicon extension are coated with epoxy. Finally, the entire assembly is coated with a 200-300 nm thick layer of parylene $N$. The long extension enables compatibility with both upright and inverted microscopes.

Optical micrographs of a finished, assembled device are presented in Figure 4. Note the long silicon extension, which enables compatibility with upright microscopes with a working distance as small as $1 \mathrm{~mm}$. The device PCB includes a pair of through holes for attachment to a three-axis micromanipulator.

\section{PROBE CHARACTERIZATION}

The high dopant concentration at the contact surface ( $\sim \mathrm{e} 20 / \mathrm{cc})$ yields Ohmic contacts. The sheet resistance $(110 \Omega / \square)$ and contact resistivity $\left(10-20 \mu \Omega-\mathrm{cm}^{2}\right)$ were calculated from Van der Pauw and Kelvin Bridge test structures and confirmed using the transfer length method on finished devices. The high contact doping both minimizes the contact resistivity and overall contact resistance of the one-dimensional transmission line. Overall, the contacts and metal interconnects contribute about $340 \Omega$ of excess resistance. In contrast, devices without the two-step diffusion and optimized metallization processes had excess resistances of 1-2 $\mathrm{k} \Omega$, severely degrading the piezoresistor force resolution.

Each silicon die contains two identical force probes. The compensation probe, set far back from the tip of the device, provides both temperature and crosstalk compensation. A Wheatstone bridge circuit transduces the resistance change of the piezoresistors into a voltage output. The main and compensation probes are placed in the bottom legs of the bridge while two potentiometers balance the top legs. It is essential to place the main and compensation probes in opposite sides of the bridge in order to compensate the actuator-sensor crosstalk during simultaneous actuation and sensing.

The Wheatstone bridge bias is generated by a $5 \mathrm{~V}$ voltage reference (ADR445), taken through a potentiometer controlled voltage divider to set the desired bias and voltage buffered. The Wheatstone bridge signal is amplified in two stages in order to maintain sufficient bandwidth. First, a low noise instrumentation 


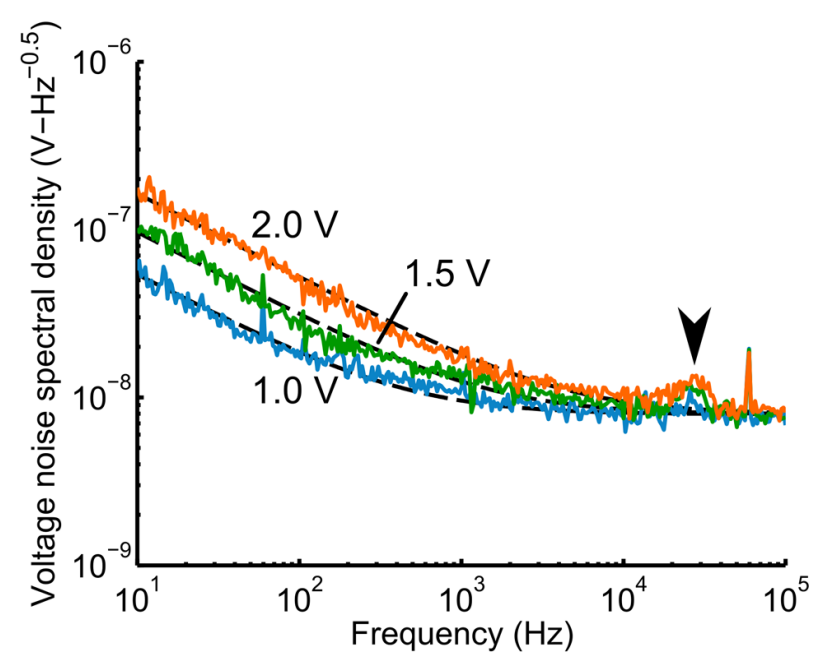

Figure 5: Example piezoresistor noise spectra in air for Wheatstone bridge bias voltages of $1,1.5$ and $2 \mathrm{~V}$. The force probe is capable of self-detecting its thermomechanical resonance in air ( $f_{0}=30 \mathrm{kHz}, Q=3.6$ ). The Hooge factor is calculated by fitting a noise model (dashed lines) to the measured spectra. For the device presented here, $\alpha=1.1 \pm 0.4 \times 10^{-5}$.

amplifier (INA103) amplifies the signal 100x $\left(\mathrm{f}_{-3 \mathrm{~dB}}=800 \mathrm{kHz}\right)$. A second-stage variable gain amplifier (THS4031) provides further gain without compromising the measurement bandwidth.

Example piezoresistor noise spectra are presented in Figure 5. The piezoresistor exhibits $1 / \mathrm{f}$ noise due to bulk mobility fluctuations. The Hooge factor $(\alpha)$ is calculated by fitting a noise model to the measured spectra. The electrically active dopant concentration profile is measured via spreading resistance analysis (Solecon Laboratories) and used to calculate the number of carriers in the piezoresistor. The effective number of carriers, accounting for the variation in current density through the depth of the piezoresistor, is about $20 \%$ smaller than the total number of carriers and is used to model the $1 / \mathrm{f}$ noise.

The piezoresistor displacement sensitivity is calibrated by applying a known deflection to the tip of the force probe. We mount the probe on a piezoelectric stage with capacitive position readout and deflect the probe with a sub-micron $\mathrm{AC}$ deflection using a stationary, stiff AFM cantilever. Example calibration data is shown in Figure 6. The measured and predicted displacement sensitivities match to within about $10 \%$. The model prediction is based upon experimentally inferred sensitivity and resistance factors $\left(\beta^{*}=0.23 \pm 5 \%, \gamma=0.83\right.$ to 0.96$)$.

Our piezoresistive sensors operate near the thermodynamic force noise limit. For example a $98 \mu \mathrm{m}$ long, $2 \mu \mathrm{m}$ wide probe has a resonant frequency in air of $28 \mathrm{kHz}$, quality factor of 3.8 and spring constant of $1.63 \pm 0.13 \mathrm{pN} / \mathrm{nm}$ (measured via thermomechanical noise). The overall force noise from $1 \mathrm{~Hz}$ to 28 $\mathrm{kHz}$ is $4.4 \pm 0.4 \mathrm{pN}$ for a $2 \mathrm{~V}$ bridge bias (to limit self-heating), compared with the thermomechanical force noise limit of $1.0 \mathrm{pN}$. Similarly results (i.e. operation at about $4 \mathrm{x}$ the thermomechanical noise floor) are obtained when the devices are operated in water.

\section{CONCLUSIONS}

We have summarized the fabrication and sensing performance of our force probes. Their force resolution, actuation speed and actuator-sensor crosstalk each represent a 10-20 fold improvement over prior devices and pave the way for $\mathrm{pN}$-scale force measurements at the microsecond time scale in water.
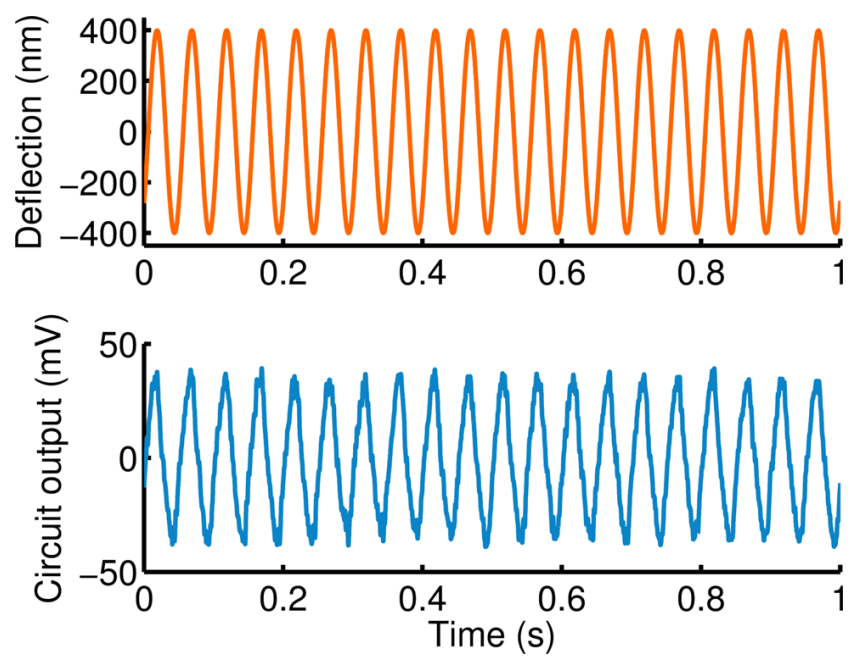

Figure 6: Example of displacement sensitivity calibration. The force probe is deflected $400 \mathrm{~nm}$ at $20 \mathrm{~Hz}$ and the output of the measurement circuit is recorded. The average displacement sensitivity for this probe was $73.5 \pm 10.3 \mathrm{~V} / \mathrm{m}$-V compared with a model prediction of $80.8 \mathrm{~V} / \mathrm{m}-\mathrm{V}$.

\section{ACKNOWLEDGEMENTS}

Fabrication work was performed at the Stanford and Lurie Nanofabrication Facilities (members of the NNIN) supported by the NSF under Grant ECS-9731293. This work was supported by the NIH under grant EB006745, the NSF under CAREER Award ECS-0449400, COINS NSF-NSEC ECS-0425914, and CPN NSFNSEC PHY-0425897, and by DARPA under YFA N66001-09-12089. JCD was supported in part by NDSEG and NSF Graduate Research fellowships. The authors are grateful to A.J. Ricci and A.W. Peng of Stanford University for their helpful input on the probe design and characterization, and S. Martin and R. Hower of the University of Michigan for performing the backside DRIE.

\section{REFERENCES}

[1] K.C. Neuman and A. Nagy, "Single-molecule force spectroscopy: optical tweezers, magnetic tweezers and atomic force microscopy", Nature Methods (2008).

[2] S.C. Minne, S.R. Manalis and C.F. Quate, "Parallel atomic force microscopy using cantilevers with integrated piezoresistive sensors and integrated piezoelectric actuators", Applied Physics Letters (1995).

[3] Y.-S. Kim et al., "PZT cantilever array integrated with piezoresistor sensor for high speed parallel operation of AFM", Sensors and Actuators A (2003).

[4] G.E. Fantner et al., "Use of self-actuating and self-sensing cantilevers for imaging biological samples in fluid", Nanotechnology (2009).

[5] J.C. Doll, S.-J. Park and B.L. Pruitt, "Design optimization of piezoresistive cantilevers for force sensing in air and water", Journal of Applied Physics (2009).

[6] J.C. Doll et al, E.A. Corbin, W.P. King and B.L. Pruitt, "Selfheating in piezoresistive cantilevers", App. Phys. Lett. (2011).

[7] G.C. Hill et al, "Patterned cracks improve yield in the release of compliant microdevices from silicon-on-insulator wafers", J. Micromech. Microeng. (2011).

\section{CONTACT}

*B.L. Pruitt; pruitt@stanford.edu 